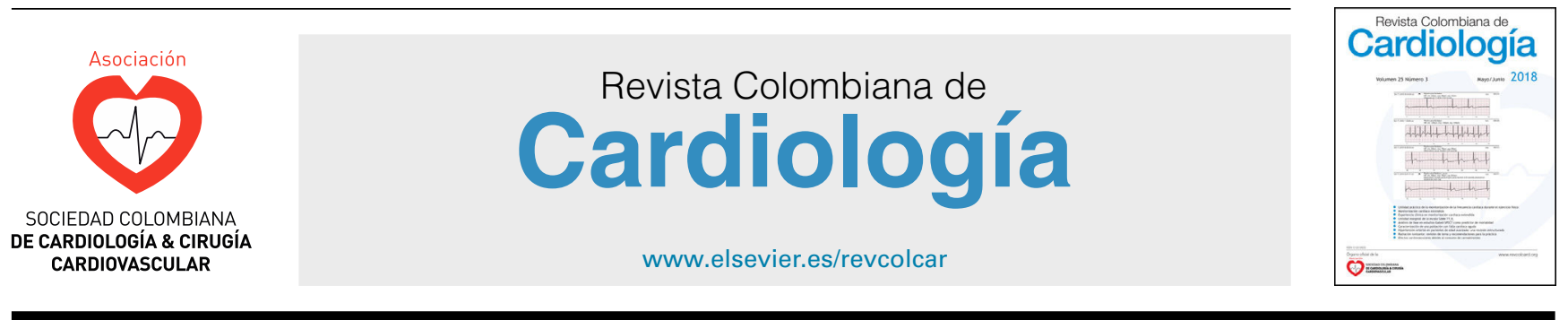

CARDIOLOGÍA DEL ADULTO - ARTÍCULO ORIGINAL

\title{
Síndrome coronario agudo en adultos jóvenes llevados a arteriografía coronaria
}

\author{
Sergio A. Higuera ${ }^{a, *}$, Laura M. Hernández-Delgado ${ }^{b}$ y Boris E. Vesga ${ }^{c}$ \\ a Departamento de Medicina Interna, Escuela de Medicina, Universidad Industrial de Santander, Bucaramanga, Colombia \\ b Fundación Cardiovascular de Colombia, Floridablanca, Colombia \\ c Departamento de Medicina Interna, Escuela de Medicina, Universidad Industrial de Santander, Instituto del Corazón de \\ Bucaramanga S.A., Bucaramanga, Colombia
}

Recibido el 21 de abril de 2018; aceptado el 24 de agosto de 2018

Disponible en Internet el 12 de diciembre de 2018

\author{
PALABRAS CLAVE \\ Infarto del miocardio; \\ Adulto joven; \\ Angiografía \\ coronaria; \\ Sobrepeso
}

\begin{abstract}
Resumen
Objetivo: describir factores de riesgo y características clínicas, electrocardiográficas y angiográficas de pacientes jóvenes que sufren síndromes coronarios agudos y son llevados a arteriografía coronaria a causa de este diagnóstico.

Métodos: estudio retrospectivo, de corte transversal. Se revisaron historias clínicas de pacientes entre 18 y 50 años, con síndrome coronario agudo en una institución de Cardiología Intervencionista en Bucaramanga.

Resultados y conclusiones: se identificaron 128 casos, con una mediana de edad de 45 años, y predominancia del género masculino $(80,4 \%)$. La mediana de tiempo a la consulta fue de 7 horas. El factor de riesgo más frecuente fue el exceso de peso ( $71,3 \%$ de los casos). La mayoría de los casos correspondió a infarto con elevación del segmento ST (44\% de los casos). En el $60,9 \%$ de los casos se identificó enfermedad coronaria, con 55,1\% de enfermedad coronaria multivaso. La intervención de reperfusión más frecuente fue la intervención coronaria percutánea mediante angioplastia coronaria con implantación de stent coronario convencional $(60,25 \%$ de los casos). La mortalidad intrahospitalaria fue del 2,34\%. El exceso de peso como principal factor de riesgo observado plantea la importancia de intervenciones poblacionales dirigidas a controlar su prevalencia. La elevada frecuencia de infartos con elevación del segmento ST y de enfermedad coronaria multivaso llama la atención sobre el riesgo de estos pacientes y la importancia de su atención temprana y diligente.

(c) 2018 Sociedad Colombiana de Cardiología y Cirugía Cardiovascular. Publicado por Elsevier España, S.L.U. Este es un artículo Open Access bajo la licencia CC BY-NC-ND (http:// creativecommons.org/licenses/by-nc-nd/4.0/).
\end{abstract}

\footnotetext{
* Corresponding author.

Correo electrónico: seanhile@hotmail.com (S.A. Higuera).
} 


\section{KEYWORDS}

Myocardial infarction;

Young adult;

Coronary

angiography;

Overweight.

\section{Acute coronary syndrome in young adults subjected to coronary angiography}

\begin{abstract}
Objective: To describe the risk factors and clinical, electrocardiographic, and angiographic characteristics of young adults that suffer from acute coronary syndromes and have been subjected to a coronary angiography due to this diagnosis.

Methods: A retrospective, cross-sectional study was performed by reviewing the clinical records of patients between 18 and 50 years-old with an acute coronary syndrome in a Interventional Cardiology institution in Bucaramanga, Colombia.

Results and conclusions: A total of 128 cases were identified, with a median age of 45 years, and the large majority $(80.4 \%)$ male. The median time in the clinic was 7 hours. The most frequent risk factor was overweight ( $71.3 \%$ of cases). The majority of cases corresponded to an infarction with elevated ST segment (44\% of cases). Coronary disease was identified in $60.9 \%$ of cases, and $55.1 \%$ with multiple vessel coronary disease. The most frequent reperfusion treatment was percutaneous coronary intervention by means of coronary angioplasty with the implantation of a conventional coronary stent $(60.25 \%$ of cases). The in-hospital mortality was $2.34 \%$. The excess weight as the main risk factor observed, shows the importance of population interventions directed at controlling its prevalence. The increased frequency of infarctions with elevated ST segment and multiple vessel coronary disease highlights the risk of these patients, and the importance of their early and rapid care.

(C) 2018 Sociedad Colombiana de Cardiología y Cirugía Cardiovascular. Published by Elsevier España, S.L.U. This is an open access article under the CC BY-NC-ND license (http:// creativecommons.org/licenses/by-nc-nd/4.0/).
\end{abstract}

\section{Introducción}

La enfermedad crónica no transmisible se ha posicionado como la principal causa de mortalidad en el mundo, mostrando una tendencia a su aumento en las últimas mediciones al respecto ${ }^{1}$. Dentro de este grupo, la cardiopatía isquémica es el principal responsable de la mortalidad, fenómeno observado también en Colombia donde $56,3 \%$ de la mortalidad en el 2011 fue causada precisamente por esta condición ${ }^{2}$. El incremento en la prevalencia de la cardiopatía isquémica se ha acompañado de un indudable incremento en el desarrollo y la publicación de estudios sobre esta entidad, lo que se ha traducido en mejoría en la atención de estos pacientes y a su vez ha reducido la mortalidad de esta enfermedad a casi una quinta parte de la que se observaba en la primera mitad del siglo $\mathrm{xx}^{3}$.

De manera llamativa se observa cada vez más síndrome coronario agudo entre pacientes jóvenes (de 50 años o menores $)^{4}$, quienes además reciben tratamiento oportuno en menos de la mitad de los $\operatorname{casos}^{5}$ y tienen un comportamiento a largo plazo en sus factores de riesgo más adverso que el de la población general ${ }^{6}$. Si partimos de la base que el síndrome coronario agudo incrementa los costos de atención en salud (desde 12.000 hasta 34.000 dólares anuales adicionales por paciente, según las comorbilidades ${ }^{7}$ ), la población jóven en particular será aún más importante por el impacto en calidad de vida y su necesaria consecuencia en productividad y carga económica a los sistemas de salud.

En Colombia existen escasos estudios sobre la población joven que sufre síndrome coronario agudo. El más grande fue publicado hace más de trece años ${ }^{8}$ y representa un momento histórico en el cual la disponibilidad de opciones terapéuticas y oportunidad en la atención eran diferentes a las de la actualidad. En vista de lo anterior, se condujo un estudio que evaluara la población jóven que sufre un síndrome coronario agudo en nuestra región para poder caracterizarla y con esta información contribuir a optimizar la prevención y el cuidado de estos pacientes, durante y después del evento agudo.

\section{Métodos}

Se realizó un estudio observacional, retrospectivo, de corte transversal en la sede Chicamocha del Instituto del Corazón de Bucaramanga S.A. Se revisaron los registros de historia clínica electrónica en búsqueda de todos los pacientes entre 18 y 50 años de edad, llevados a arteriografía coronaria entre el $1^{\circ}$. de enero de 2009 y el 31 de diciembre del 2014, con diagnósticos por códigos CIE-10 que pudieran ser compatibles con síndrome coronario agudo (todos los comprendidos entre I20X-125X y Z955). Una vez obtenida esta selección preliminar de pacientes se revisaron las historias clínicas para verificar el lugar de atención, el diagnóstico clínico de síndrome coronario agudo y la realización de arteriografía coronaria a raíz de dicho diagnóstico, y se determinó la inclusión de pacientes a la base de datos.

Se exploraron cuatro grupos de variables: demográficas (edad y género), clínicas (manifestaciones de ingreso, incluyendo las electrocardiográficas, fracción de eyección ventricular izquierda, complicaciones durante la hospitalización, tiempo al egreso y mortalidad intrahospitalaria), factores de riesgo y angiográficas (enfermedad coronaria, intervenciones coronarias percutáneas y otros hallazgos angiográficos). El análisis estadístico fue descriptivo univariado con proporciones para variables categóricas y nominales. Las variables cuantitativas se evaluaron con la 
prueba de Shapiro-Wilk para determinar si presentaban comportamiento normal y según esto reportar promedios con desviaciones estándar o medianas con rangos intercuartílicos respectivamente. Se realizaron análisis exploratorios bivariados mediante la prueba de $X^{2}$ (prueba exacta de Fisher cuando las tablas de contingencia formaban grupos menores de 5 sujetos) para la comparación de prevalencia de factores de riesgo entre géneros y según la presencia o ausencia de enfermedad coronaria. La comparación de edad, tiempo de síntomas e índice de masa corporal entre los grupos mencionados se realizó mediante la prueba de Mann-Whitney-Wilcoxon (por su distribución no normal). Todos los análisis se hicieron con el paquete estadístico STATA/MP 13.0 para macOS.

El estudio se diseñó en concordancia con los lineamientos de Buenas Prácticas Clínicas del Comité Internacional de Armonización y los principios éticos de la Declaración de Helsinki. El protocolo de investigación fue sometido y aprobado por el Comité de Ética e Investigaciones del Instituto del Corazón de Bucaramanga S.A. previo al inicio de los procedimientos del estudio para garantizar la integridad ética del mismo.

\section{Resultados}

Se identificaron un total de 533 potenciales pacientes en quienes se evaluaron historias clínicas completas para determinar elegibilidad, incluyendo finalmente al estudio 128 casos. Las evaluaciones de normalidad de las variables continuas: edad, tiempo al ingreso a Urgencias, índice de masa corporal, fracción de eyección, número de stent utilizados y tiempo al egreso hospitalario mostró un comportamiento anormal (prueba de Shapiro-Wilk <0,001 para todas las evaluaciones) por lo que se reportan como medianas con rangos intercuartílicos.

Se observó preponderancia marcada del sexo masculino, con un $80,5 \%(n=103)$ correspondiente a hombres y el restante $19,5 \%$, a mujeres $(n=25)$. La mediana de edad fue de 45 años (rango intercuartílico 41,5 a 48 años), mayor en mujeres (47 años) que en hombres (44 años) de manera estadísticamente significativa $(p=0,0492)$. El tiempo de consulta al servicio de Urgencias tuvo una mediana de 7 horas (rango intercuartílico 2 - 24 horas), sin diferencias estadísticamente significativas entre géneros $(p=0,4433)$. Respecto a las ventanas de interés terapéutico se encontró que para la hora seis de síntomas ya habían consultado el 49,2\% de los pacientes $(n=63)$, para la hora 12 el $61,7 \%(n=79)$ y para la hora 48 el $89,1 \%(n=114)$.

El síntoma más común fue el dolor torácico característico (opresivo, de ubicación precordial, relacionado con el estrés físico/emocional y que cedía en el reposo/con nitratos), presente en un $89,8 \%(n=115)$. La diaforesis y la disnea fueron los siguientes síntomas en frecuencia, presentes en aproximadamente un tercio de los pacientes cada uno. Otras manifestaciones clínicas fueron menos comunes, con tan solo 2 pacientes $(1,16 \%)$ con parada cardiorrespiratoria al ingreso a urgencias (para una descripción completa de los síntomas observados en los pacientes ver la tabla 1). La clasificación de Killip-Kimball mostró un predominio de la clase I, observada en el 92,2\% $(n=118)$. El 2,3\% $(n=3)$ se ubicaba
Tabla 1 Frecuencia de los síntomas evaluados al ingreso a Urgencias

\begin{tabular}{ll}
\hline Síntoma & Prevalencia $(n)$ \\
\hline Dolor torácico característico & $89,84 \%(n=115)$ \\
Diaforesis & $36,72 \%(n=47)$ \\
Disnea & $29,69 \%(n=38)$ \\
Dolor torácico no característico & $7,03 \%(n=9)$ \\
Palpitaciones & $6,25 \%(n=8)$ \\
Disminución de clase funcional & $6,25 \%(n=8)$ \\
Síncope extrahospitalario & $5,47 \%(n=7)$ \\
Taquicardia & $2,34 \%(n=3)$ \\
Parada cardiorrespiratoria & $1,56 \%(n=2)$ \\
\hline
\end{tabular}

en clase II, el $1,6 \%(n=2)$ en clase III y un $3,9 \%(n=5)$ en clase IV.

En la tabla 2 se resumen los factores de riesgo identificados en los pacientes. En los factores de riesgo tradicionales solamente el consumo de tabaco mostró diferencias estadísticamente significativas entre hombres y mujeres, que fue mayor en los primeros $(36,8 \%, n=38)$ que en las segundas $(12,0 \%, n=3)$. El índice de masa corporal tuvo una mediana de $26,6 \mathrm{~kg} / \mathrm{m}^{2}$ (rango intercuartílico 24,3 a $29,7 \mathrm{~kg} / \mathrm{m}^{2}$ ), sin diferencias estadísticamente significativas entre sexos $(p=0,2365)$. El exceso de peso fue el principal factor de riesgo identificado en la población, con un $71,3 \%$ de los pacientes ( $n=82$ de los 115 con datos sobre peso y talla para cálculo del índice) e índice de masa corporal igual o superior a $25 \mathrm{~kg} / \mathrm{m}^{2}$; así mismo un $23,47 \%$ cursaba con algún grado de obesidad. El $28,1 \%(n=36)$ tenía exceso de peso como único factor de riesgo identificado. Sólo un paciente fue negativo para todos los factores de riesgo.

En cuanto al consumo de sustancias, la prevalencia más elevada fue para la historia de consumo de alcohol observada en un $11,7 \%$ de los casos $(n=15)$. El consumo de cocaína o productos derivados se identificó en tan solo 4 casos $(3,1 \%)$. Previo al inicio de la sintomatología, 2 pacientes refirieron la administración de esteroides anabólicos autoprescritos para el aumento de masa muscular, aunque ninguno de ellos especificó agente, posología ni historia previa de uso de dichas sustancias. En los casos revisados no se identificó historia de consumo de otras sustancias.

Todos tenían al menos un electrocardiograma de ingreso a Urgencias (en los 2 pacientes con parada cardiorrespiratoria se obtuvo posterior al retorno a circulación espontánea). El principal ritmo de base identificado fue el sinusal, en 96,1\% $(n=123)$; un 9,38\% ( $n=12)$ mostraba taquicardia sinusal. Los 5 pacientes restantes tenían ritmo de bloqueo auriculoventricular: $2(1,6 \%)$ de grado I, uno $(0,8 \%)$ de grado II y 2 $(1,6 \%)$ de grado III. No se identificaron otros ritmos de base. El 3,9\% tenía bloqueo de rama derecha $(n=5)$ y apenas el $0,8 \%$ bloqueo de rama izquierda $(n=1)$. No se hallaron otras manifestaciones electrocardiográficas, incluidas taquicardia ventricular o ritmo de fibrilación ventricular.

La distribución entre los distintos tipos de síndrome coronario agudo mostró predominio del síndrome coronario agudo con elevación del segmento ST, el cual se observó en poco más de un tercio de los pacientes (fig. 1). En aquellos con elevación del segmento ST la afectación más frecuente fue la de la pared inferior, en el $42,86 \%(n=24)$ (tabla 3$)$. 
Tabla 2 Factores de riesgo identificados

\begin{tabular}{|c|c|c|c|c|}
\hline \multirow[t]{2}{*}{ Factor de riesgo } & \multicolumn{3}{|c|}{ Prevalencia (n) } & \multirow[t]{2}{*}{$P^{* *}$} \\
\hline & General & Hombres & Mujeres & \\
\hline Hipertensión arterial & $35,9 \%(46)$ & $33,0 \%(34)$ & $48,0 \%(12)$ & 0,161 \\
\hline Tabaquismo (actual o suspendido) & $32,0 \%(41)$ & $36,8 \%(38)$ & $12,0 \%(3)$ & 0,017 \\
\hline Dislipidemia (cualquier tipo) & $20,3 \%(26)$ & $22,3 \%(23)$ & $12,05(3)$ & 0,405 \\
\hline Diabetes mellitus & $14,8 \%(19)$ & $13,6 \%(14)$ & $20,0 \%(5)$ & 0,419 \\
\hline Antecedente personal de enfermedad coronaria & $10,2 \%(13)$ & $9,7 \%(10)$ & $12,0 \%(3)$ & 0,134 \\
\hline$I M C\left(\mathrm{~kg} / \mathrm{m}^{2}\right)$ & $26,6(24,3-29,7)^{*}$ & $26,8(24,7-29,7)^{*}$ & $25,4(23,6-29,6)^{*}$ & 0,2365 \\
\hline
\end{tabular}

" Medianas (rangos intercuartílicos) en los sujetos con disponibilidad de dato sobre índice de masa corporal.

** Valor de $p$ para evaluación de significancia estadística de las diferencias entre hombres y mujeres en los análisis exploratorios.

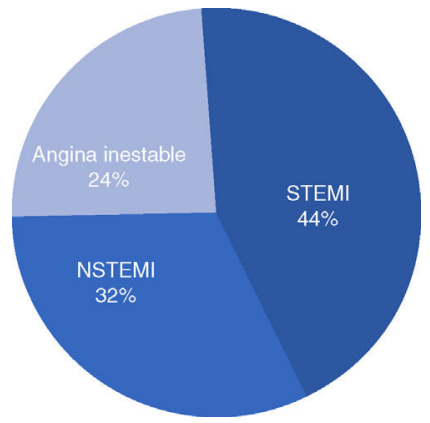

Figura 1 Distribución entre los distintos tipos de síndrome coronario agudo.

Tabla 3 Paredes afectadas en el electrocardiograma de ingreso

\begin{tabular}{ll}
\hline Pared afectada & Prevalencia $(n)^{*}$ \\
\hline Pared septal & $12,50 \%(n=7)$ \\
Pared anterior & $14,29 \%(n=8)$ \\
Pared anteroseptal & $17,86 \%(n=10)$ \\
Pared lateral & $12,50 \%(n=7)$ \\
Pared anterolateral & $12,50 \%(n=7)$ \\
Pared inferior & $42,86 \%(n=24)$ \\
Extensión al ventrículo derecho & $1 \%(n=1,79)$ \\
\hline
\end{tabular}

* Calculada sobre el total de pacientes con enfermedad coronaria

La fracción de eyección ventricular izquierda tuvo una mediana de 55\% (rango intercuartílico de 43 a 62\%), con un valor mínimo de $20 \%$ identificado en uno de los pacientes. La mayoría $(57,99 \%, n=69)$ tenía fracción de eyección preservada. El 42,01\% $(n=50)$ tenía fracción de eyección menor o igual del $50 \%$, el $15,96 \%(n=19)$ menor o igual del $40 \%$ y tan solo el $3,36 \%(n=4)$ menor o igual al $30 \%$.

\section{Hallazgos angiográficos}

La dominancia coronaria fue derecha en $89,84 \%$ de los casos $(n=115)$. El $60,94 \%(n=78)$ de los casos estudiados tuvo enfermedad coronaria aterosclerótica obstructiva de al menos un vaso. La distribución de la frecuencia de compromiso de cada uno de los distintos vasos principales puede
Tabla 4 Distribución del compromiso de los distintos vasos principales

\begin{tabular}{ll}
\hline Vaso afectado & Prevalencia* $(n)$ \\
\hline Coronaria derecha & $65,38 \%(n=51)$ \\
Descendente anterior izquierda & $64,10 \%(n=50)$ \\
Circunfleja izquierda & $41,03 \%(n=32)$ \\
Ramus intermedio & $12,82 \%(n=10)$ \\
Tronco principal de la coronaria izquierda & $1,28 \%(1)$ \\
\hline${ }^{*}$ Calculada sobre el total de pacientes con enfermedad & \\
coronaria &
\end{tabular}

observarse en la tabla 4. La enfermedad coronaria multivaso fue la forma más observada, en $55,13 \%$ de los casos ( $n=43)$; una quinta parte $(20,51 \%, n=16)$ de los casos mostró afectación de los tres vasos principales.

El tratamiento de reperfusión se hizo mediante intervención coronaria percutánea primaria, con uso marginal de la trombólisis en 4 casos $(3,12 \%)$. El 80,72\% $(n=111)$ de los pacientes fueron llevados a algún tipo de intervención coronaria percutánea (incluye los procedimientos hechos en pacientes sin enfermedad coronaria), con un total de 72 lesiones ateroscleróticas obstructivas tratadas con stent coronarios en 65 intervenciones coronarias percutáneas distintas. Respecto a los dispositivos intracoronarios, el más utilizado fue el stent coronario convencional en $60,25 \%$ de las lesiones $(n=47)$, seguido del stent coronario medicado en $29,48 \%(n=23)$. En 2 casos $(2,56 \%)$ se realizó tratamiento únicamente mediante angioplastia coronaria con balón sin implantación de stent intracoronario, correspondiendo en ambas ocasiones a imposibilidad de posicionamiento adecuado del stent durante el procedimiento, para el tratamiento de la lesión objetivo.

El vaso más tratado fue la coronaria derecha, involucrada en $52,3 \%(n=34)$ de los procedimientos de intervención coronaria percutánea, seguida muy de cerca de la descendente anterior, involucrada en $49,2 \%(n=32)$ de los procedimientos. Se hizo intervención coronaria percutánea multivaso en $29,23 \%$ del total de procedimientos $(n=19)$. Se hizo revascularización quirúrgica del miocardio en apenas 7 pacientes $(8,97 \%)$ del total de aquellos con enfermedad coronaria aterosclerótica obstructiva.

Se observaron complicaciones derivadas del procedimiento en el $3,12 \%$ de los casos $(n=4)$, correspondiendo 
Tabla 5 Características clínicas, demográficas y de factores de riesgo en el grupo de pacientes sin enfermedad coronaria aterosclerótica obstructiva

\begin{tabular}{lll}
\hline Característica & Prevalencia $(\mathrm{n})$ & $P^{*}$ \\
\hline Dolor torácico característico & $88 \%(\mathrm{n}=44)$ & 0,580 \\
Dolor torácico no característico & $10 \%(\mathrm{n}=5)$ & 0,293 \\
Disnea & $26 \%(\mathrm{n}=13)$ & 0,465 \\
Disminución de clase funcional & $8 \%(\mathrm{n}=4)$ & 0,513 \\
Hipertensión arterial & $24 \%(\mathrm{n}=12)$ & 0,024 \\
Diabetes mellitus & $12 \%(\mathrm{n}=6)$ & 0,469 \\
Dislipidemia & $10 \%(\mathrm{n}=5)$ & 0,016 \\
Tabaquismo & $14 \%(\mathrm{n}=7)$ & 0,000 \\
Enfermedad renal crónica & $6 \%(\mathrm{n}=3)$ & 0,563 \\
IMC (kg/m²) & $25,0(23,6-29,6)$ & 0,0795 \\
\hline
\end{tabular}

* Valor de $p$ para evaluación de significancia estadística de diferencias con el grupo de pacientes con enfermedad coronaria obstructiva en los análisis exploratorios

*** Mediana con rango intercuartílico estimada sobre los 45 casos con disponibilidad de dato de índice de masa corporal.

en todos ellos a hematomas del sitio de punción (todas punciones femorales). Las complicaciones intrahospitalarias incluyeron 3 casos de arritmias estables, un caso de arritmia inestable con requerimiento de cardioversión, un caso de arritmia fatal, 2 casos de shock cardiogénico persistente, un caso de complicación mecánica del infarto y 3 casos de lesión renal aguda. Se evidenció mortalidad intrahospitalaria en 3 casos para una tasa del 2,34\%. Estas 3 muertes correspondieron a uno de los pacientes con shock cardiogénico persistente, el paciente que presentó una complicación mecánica (insuficiencia mitral isquémica aguda) y el paciente con la arritmia fatal (fibrilación ventricular). El tiempo al egreso hospitalario tuvo una mediana de cuatro días, con un rango intercuartílico de dos a cinco días y sin diferencias significativas entre géneros $(p=0,5372)$.

\section{Pacientes sin enfermedad coronaria}

En el $39,06 \%$ de los casos $(n=50)$ no se identificaron lesiones coronarias ateroscleróticas obstructivas. En la tabla 5 se resumen las características clínicas, demográficas y de factores de riesgo observadas en esta población. La mediana de edad fue de 43 años (rango intercuartílico 35 - 47 años), siendo menor en comparación con el grupo de pacientes con enfermedad coronaria de manera estadísticamente significativa $(p=0,0031)$. Al igual que en la población general se observó mayor prevalencia del género masculino $(74 \%$, $\mathrm{n}=37)$. En comparación con el grupo de pacientes con enfermedad coronaria hubo una prevalencia menor de hipertensión arterial, dislipidemia y consumo de tabaco.

El predominio de los casos de infarto de miocardio con elevación del ST observados en la población general del estudio no se observó entre los pacientes sin enfermedad coronaria: $32 \%(n=16)$ de los casos correspondieron a anginas inestables y un $34 \%(n=17)$ para cada grupo de infarto con y sin elevación del segmento ST. En lo que respecta a las observaciones electrocardiográficas también se observó diferencia en cuanto a la pared más afectada, siendo esta la anterior $(n=10)$ y no la inferior $(n=6)$. La mediana de fracción de eyección fue de $60 \%$ (rango intercuartílico $50-63 \%)$, con compromiso de la fracción de eyección (<50\%) en tan solo 4 pacientes ( $8 \%$ del grupo).

En lo que concierne a la angiografía, el $14 \%$ de los pacientes $(n=7)$ tenía imágenes de trombosis coronaria sin lesiones ateroscleróticas visibles por angiografía (en los pacientes con enfermedad coronaria no hubo ningún caso de estas características), $6 \%(n=3)$ de los cuales requirieron angioplastia coronaria con balón para la restauración del flujo coronario distal. Respecto a complicaciones se identificaron dos hematomas en el sitio de punción y una lesión renal aguda asociada al medio de contraste. No hubo ningún caso de mortalidad intrahospitalaria. El tiempo al egreso hospitalario tuvo una mediana de dos días (rango intercuartílico uno a tres días), el cual fue significativamente más corto $(p=0,0000)$ que en los pacientes con enfermedad coronaria (mediana de cuatro días con rango intercuartílico de tres a siete días.

\section{Discusión}

Varios resultados del estudio merecen ser comentados por su importancia clínica o para futuros proyectos de investigación. En primera instancia se resalta el tiempo a la consulta médica, la cual es una de las demoras en la atención clínica ${ }^{9}$, que impacta en la disponibilidad de opciones de tratamiento para los pacientes. Una amplia mayoría consultó en una ventana de tiempo adecuada para ofrecer tratamiento con intervención coronaria percutánea primaria, lo cual a su vez enfatiza la importancia de reconocer a los pacientes candidatos a esta intervención y derivarlos de la manera más oportuna posible a los laboratorios de Hemodinamia.

En lo referente a los factores de riesgo, existen tres puntos que precisan mención. El primero de ellos es la prevalencia elevada de factores de riesgo tradicionales (la hipertensión y el tabaquismo se observaron cada uno en alrededor de un tercio de los pacientes), hecho que denota las consecuencias de la transición epidemiológica que vive nuestro país ${ }^{10}$ y que previsiblemente continuará aumentando la prevalencia de estos factores de riesgo.

El segundo punto es la elevada prevalencia del sobrepeso y la obesidad que en su conjunto estaban presentes en más de dos tercios de la población y se constituyen de esta manera en el principal factor de riesgo identificado. Los resultados preliminares de la Encuesta Nacional de Situación Nutricional 2015 recientemente conocidos ${ }^{11}$ mostraron una prevalencia del exceso de peso del $56,4 \%$ en la población adulta colombiana lo cual representa un aumento de más de cinco puntos porcentuales en comparación con la medición previa del 2010 y que marca una preocupante tendencia. Estos hallazgos pueden a su vez considerarse una oportunidad para reevaluar las estrategias que a nivel individual utilizamos en nuestra práctica clínica diaria para incentivar el mantenimiento de un peso normal en los pacientes; y a nivel de sistema en los programas de promoción y prevención que buscan controlar el exceso de peso, especialmente en poblaciones jóvenes "sanas" antes de que ocurran los desenlaces clínicos.

Como último punto, respecto a los factores de riesgo es cuando menos interesante los casos de síndrome coronario 
agudo relacionados con el uso de esteroides anabolizantes. Aunque el síndrome coronario agudo no es una de las consecuencias clínicas más sospechadas u observadas en los usuarios de estas sustancias, existen varios reportes al respecto ${ }^{12-14}$, que las plantean como posibles factores implicados en el desarrollo de policitemia ${ }^{12}$ e incremento en la producción de tromboxano $\mathrm{A}_{2}{ }^{13}$.

Dentro de los hallazgos angiográficos uno de los más relevantes es la elevada frecuencia de enfermedad coronaria multivaso, que representa un poco más de un tercio de los pacientes con enfermedad coronaria. Esta mayor severidad plantea la posibilidad de un largo tiempo de exposición a factores de riesgo, así como mayor probabilidad de desenlaces clínicos adversos en términos de deterioro de la función ventricular y nuevos eventos coronarios ${ }^{15}$. Aunque no se evaluó en este estudio, en futuros estudios deberían incluirse evaluaciones genéticas dirigidas a determinar si existen polimorfismos que potencien un estado pro-aterogénico y expliquen los hallazgos observados.

El grupo de pacientes sin enfermedad coronaria muestra algunas diferencias en ciertas características clínicas, que en los análisis exploratorios sugieren ser de significancia estadística. Futuros estudios dirigidos de manera específica a este grupo de pacientes podrían dilucidar factores que puedan ayudar a predecir la ausencia de enfermedad coronaria en pacientes con infarto de miocardio.

\section{Limitaciones y fortalezas}

La dos limitaciones principales son la naturaleza retrospectiva (lo cual limita la disponibilidad de información a lo ya evaluado y consignado en las historias clínicas) y la definición de los factores de riesgo basada en el reporte de los mismos en las historias clínicas y no en mediciones directas. Aunque importantes, estas dos limitaciones se observan en la amplia mayoría de estudios dirigidos a esta población; por tanto, a partir de esta observación se plantea la necesidad de proyectos prospectivos futuros que subsanen esta debilidad. En la selección de pacientes se tomaron solamente aquellos con síndrome coronario agudo, por lo cual llegan a arteriografía coronaria, de modo que estos resultados no son extrapolables a una población más general (por ejemplo, de consultas a Urgencias por dolor torácico). En el apartado de factores de riesgo no se contó con información sobre historia familiar de enfermedad coronaria y sedentarismo en la mayoría de las historias, por lo que se decidió no incluirlos en el análisis.

Así como limitaciones, existen algunas fortalezas que se pueden destacar. Al revisar los estudios latinoamericanos sobre esta población puede decirse que este estudio es el más grande de los recientemente publicados. Los análisis exploratorios sugieren la presencia de diferencias entre grupos de interés, lo cual a su vez plantea hipótesis para exploración en futuros estudios de esta población. El ámbito en el cual se llevó a cabo el estudio (una institución de referencia en la atención de tercer nivel de pacientes con síndrome coronario agudo) provee robustez sobre la calidad de los datos, especialmente en lo referente a la evaluación cardiológica (electrocardiográfica, ecocardiográfica y angiográfica).

\section{Conclusiones}

Este estudio demuestra como los síndromes coronarios agudos en adultos jovenes cursan con caracteríticas diferenciales importantes por el riesgo asociado, tales como la mayor incidencia de casos con elevación del segmento ST o de enfermedad coronaria multivaso. Así mismo, la elevada prevalencia del exceso de peso es alarmante por la carga de enfermedad asociada pero puede también considerarse una oportunidad de intervención en salud pública. Algunos de los análisis bivariados exploratorios sugieren diferencias significativas entre géneros y entre los pacientes con y sin enfermedad coronaria, las cuales, de verificarse en otros estudios, deben tenerse en cuenta al momento de diseñar los planes de prevención secundaria a largo plazo de estos pacientes. Se requieren futuros estudios prospectivos que de manera sistemática evalúen esta población, incluyendo evaluaciones de factores de riesgo no convencional, perfilamiento genético e identificación de factores clínicos predictores de enfermedad coronaria.

\section{Financiación}

Ninguna.

\section{Conflicto de intereses}

Ninguno.

\section{Agradecimientos}

A la Unidad de Investigaciones y Educación Médica del Instituto del Corazón de Bucaramanga por su apertura y colaboración para el desarrollo de este estudio.

\section{Bibliografía}

1. World Health Organization. Global Status Report On Noncommunicable Diseases 2014. Ginebra: World Health Organization; 2014.

2. Observatorio Nacional de Salud. Boletín No. 1, Diciembre 9 de 2013 - Enfermedad cardiovascular: principal causa de muerte en Colombia. Bogotá D.C.: Instituto Nacional de Salud; 2013.

3. Nabel EG, Braunwald E. A tale of coronary artery disease and myocardial infarction. N Engl J Med. 2012;366:54-63.

4. Gupta A, Wang Y, Spertus JA, Geda M, Lorenze N, Nkonde-Price $C$, et al. Trends in acute myocardial infarction in young patients and differences by sex and race, 2001 to 2010. J Am Coll Cardiol. 2014;64:337-45.

5. Pelletier R, Humphries KH, Shimony A, Bacon SL, Lavoie KL, Rabi D, et al. Sex-related differences in access to care among patients with premature acute coronary syndrome. Can Med Assoc J. 2014;186:497-504.

6. Leung Yinko SS, Maheswaran J, Pelletier R, Bacon SL, Daskalopoulou SS, Khan NA, et al. Sex differences in health behavior change after premature acute coronary syndrome. Am Heart J. 2015;170:242-8.

7. Ghushchyan V, Nair KV, Page RL. Indirect and direct costs of acute coronary syndromes with comorbid atrial fibrillation, heart failure, or both. Vasc Health Risk Manag. 2014;11:25-34. 
8. Marín F, Ospina LF. Infarto agudo del miocardio en adultos jov́enes menores de 45 años. Rev Colomb Cardiol. 2004;11:193-204.

9. Ibanez B, James S, Agewall S, Antunes MJ, Bucciarelli-Ducci C, Bueno H, et al. 2017 ESC Guidelines for the management of acute myocardial infarction in patients presenting with STsegment elevation. Eur Heart J. 2018;39:119-77.

10. Ramos-Clason EC. Transición epidemiológica en Colombia: de las enfermedades infecciosas a las no transmisibles. Rev Cienc Biomed. 2012;3:282-90.

11. Gaviria A. Resultados de la encuesta ENSIN sobre situación nutricional. 2017 Nov 20 [citado 2017 Dic 10]. En: Alejandro Gaviria [Internet]. Bogotá D.C.: Alejandro Gaviria. Disponible en: agaviria.blogspot.com/2017/11/resultados-dela-encuesta-ensin-sobre.html/.

12. Stergiopoulos K, Brennan JJ, Mathews R, Setaro JF, Kort S. Anabolic steroids, acute myocardial infarction and polycythemia: a case report and review of the literature. Vasc Health Risk Manag. 2008;4:1475-80.

13. Pontes Santos R, Pereira A, Guedes H, Lourenço C, Azevedo J, Pinto P. Anabolic drugs and myocardial infarction: A clinical case report. Arq Bras Cardiol. 2015;105:316-9.

14. Major RW, Pierides M, Squire IB, Roberts E. Bodybuilding, exogenous testosterone use and myocardial infarction. QJM. 2015; 108:651-2.

15. Muller DW, Topol EJ, Ellis SG, Sigmon KN, Lee K, Califf RM. Multivessel coronary artery disease: a key predictor of short-term prognosis after reperfusion therapy for acute myocardial infarction. Thrombolysis and Angioplasty in Myocardial Infarction (TAMI) Study Group. Am Heart J. 1991;121 4 Pt 1: 1042-9. 